\title{
mRCC: Wann Therapieabbruch wegen Toxizität?
}

\author{
In einer Studie wurden Risikofaktoren für Toxizitäten unter Anti-VEGF- \\ Therapien bei Patienten mit mRCC gesucht und außerdem ein Modell zur \\ Vorhersage toxizitätsbedingter Therapieabbrüche entwickelt.
}

nhibitoren des VEGF(„,vascular endothelial growth factor")-Signalwegs sind Standardtherapien beim metastasierten Nierenzellkarzinom (mRCC), aber Toxizitäten können zu Dosisreduktionen, Therapieunterbrechungen oder -abbrüchen führen und damit die Prognose be-

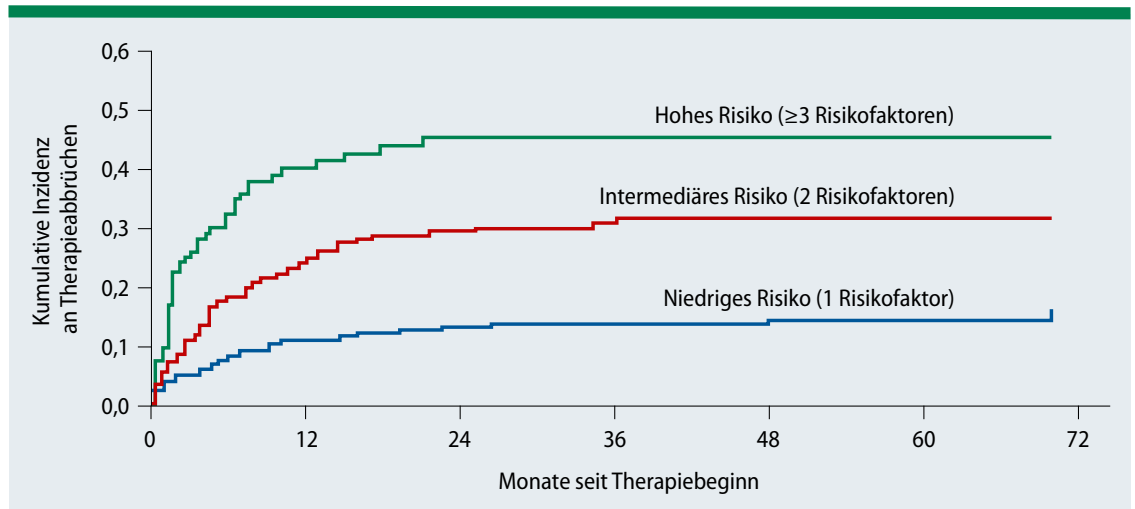

Abb.: Das Risiko für einen Therapieabbruch steigt mit der Zahl der Risikofaktoren.

\section{Niedrig dosiertes Morphin vs. schwaches Opioid}

Reicht es aus, unter moderaten Schmerzen leidende onkologische Patienten mit einem schwachen Opioid zu behandeln, oder sollten sie besser niedrig dosiertes Morphin erhalten? Dieser Frage gingen Forscher aus Italien nach.

Es mehren sich die Hinweise, dass starZke Opioide schon bei moderaten Schmerzen vorteilhafter sein könnten.

In einer Phase-III-Studie wurde bei opioidnaiven Patienten mit moderaten Tumorschmerzen eine Therapie mit niedrig dosiertem Morphin oder einem standarddosierten schwachen Opioid verglichen. 240 erwachsene Krebspatienten wurden auf das niedrig dosierte Morphin (M-Gruppe; $\mathrm{n}=118$ ) oder ein schwaches Opioid (WO-Gruppe; $\mathrm{n}=122$ ) über 28 Tage randomisiert. Primärer Endpunkt war das Ansprechen, das heißt ein Rückgang der Schmerzintensität um $20 \%$ auf einer numerischen Skala.
88,2\% der M-Gruppe und 57,7\% der WO-Gruppe erreichten dieses Ziel $(\mathrm{p}<0,001)$. Eine klinisch bedeutsame $(\geq 30 \%)$ und hoch bedeutsame Schmerzreduktion $(\geq 50 \%)$ im Vergleich zu Studienbeginn wurden signifikant häufiger unter niedrig dosiertem Morphin erreicht $(\mathrm{p}<0,001)$. Weil die Schmerztherapie nicht befriedigend war, wechselten 41 Patienten (35\%) der WO-Gruppe auf ein starkes Opioid und 17 Patienten (15,5\%) der M-Gruppe auf ein anderes starkes Opioid ( $\mathrm{p}=0,001) .28,2 \%(33 / 117) \mathrm{der}$ WO- und 13,6\% (15/110) der M-Gruppe benötigten eine Dosissteigerung ( $\mathrm{p}=$ 0,007). Der Opioid-Eskalations-Index samt 7,1 Monate und 4,4 Monate bei toxizitätsbedingten Therapieabbrüchen.

833 Patienten hatten die Therapie abgebrochen, $23,8 \%$ wegen Toxizitäten. Die kumulative 1-Jahres-Inzidenz dafür betrug $17 \%$ unter Sunitinib und $23 \%$ unter Sorafenib. Häufigste zu Therapieabbrüchen führende Toxizitäten waren Fatigue (4\%), Diarrhö (2\%) und Appetitmangel (2\%). In einer multivariaten Analyse waren vier Faktoren prädiktiv für Abbrüche: glomeruläre Filtrationsrate $($ GFR $)<30 \mathrm{ml} / \mathrm{min} / 1,73 \mathrm{~m}^{2}$ $(\mathrm{p}=0,122)$, solitäre Metastasen $(\mathrm{p}=0,096)$, Natriumspiegel $<135$ $\mathrm{mmol} / \mathrm{l}(\mathrm{p}=0,0056)$ und Alter $\geq 60$ Jahre $(\mathrm{p}=0,0022)$. In einem Vorhersagemodell hing das Abbruchrisiko von der Zahl der Risikofaktoren ab (Abb.).

Fazit: Bei der Anti-VEGF-Therapie des mRCC sind Alter, GFR, Metastasenzahl und Natriumspiegel unabhängige Risikofaktoren für Abbrüche. Judith Neumaier

Kaymakcalan MD et al. Risk Factors and Model for Predicting Toxicity-Related Treatment Discontinuation in Patients With Metastatic Renal Cell Carcinoma Treated With Vascular Endothelial Growth Factor-Targeted Therapy: Results From the International Metastatic Renal Cell Carcinoma Database Consortium. Cancer. 2016;122(3):411-9.

war in der M-Gruppe niedriger als in der WO-Gruppe $(4,76$ vs. 8,76; $\mathrm{p}=0,002)$.

Gemessen am Gesamtsymptomwert im Edmonton Symptom Assessment System war die allgemeine Verfassung der Patienten in der M-Gruppe besser als in der WO-Gruppe. Die Nebenwirkungen waren in beiden Gruppen vergleichbar.

Fazit: Krebspatienten mit moderaten Schmerzen kann offenbar bei insgesamt ähnlich guter Verträglichkeit mit niedrig dosiertem Morphin besser geholfen werden als mit schwachen Opioiden. Alternativ zum 3-Stufen-Plan der WHO könnte man womöglich einen 2-Stufen-Plan mit Verzicht auf schwache Opioide etablieren. Hierfür wären jedoch Phase-IIIb/ IV-Studien nötig. Kathrin von Kieseritzky

Bandieri E et al. Randomized Trial of Low-Dose Morphine Versus Weak Opioids in Moderate Cancer Pain. J Clin Oncol. 2016;34(5):436-42. 